\title{
SARS-CoV-2 infection after alemtuzumab in a multiple sclerosis patient: milder disease symptoms in comparison with coinfected relatives: a case report and review of the literature
}

\author{
Lorenzo Saraceno $^{1}$ (D) Emanuela Laura Susani ${ }^{1}$ • Maria Raffaella Marazzi ${ }^{1}$ • Maria Cristina Moioli ${ }^{2}$. \\ Elio Clemente Agostoni ${ }^{1} \cdot$ Alessandra Protti $^{1}$
}

Received: 15 April 2021 / Accepted: 14 August 2021 / Published online: 24 August 2021

(C) Fondazione Società Italiana di Neurologia 2021

\begin{abstract}
Literature data reporting SARS-CoV-2 infection in multiple sclerosis (MS) patients recently treated with immunodepleting agents as cladribine and alemtuzumab are very limited. The relationship between iatrogenic immunodeficiency and risk related to SARS-CoV-2 infection and its severe complications is still not clear. Cautiously, the start of immunosuppressant drugs as alemtuzumab and cladribine during the current COVID-19 pandemic is not recommended unless treatment benefits significantly outweigh potential risks. We report the case of a 30-year-old female MS patient infected by SARS-CoV-2 virus 4 months after alemtuzumab II cycle, while she was still leukopenic and lymphopenic. She had no complications and also presented milder COVID-related signs and symptoms as compared to her coinfected relatives (father, mother and her partner). Anti-S1 and S2 SARS-CoV-2 antibodies, tested 1 month and a half after the infection, resulted positive. We review all cases reported in literature of SARS-CoV-2 infection in MS patients treated with alemtuzumab. None of them had complications or severe disease.
\end{abstract}

Keywords Multiple sclerosis $\cdot$ SARS-CoV-2 $\cdot$ Coronavirus $\cdot$ Alemtuzumab $\cdot$ Cladribine $\cdot$ Immunodepleting agents

\section{Introduction}

The COVID-19 pandemic in 2020 opened an unexpected scenario for neurologists treating MS. Obesity, advanced age, pulmonary diseases, male gender and acquired lymphopenia after viral infection are known risk factors for the disease clinical severity [1]. However, to date, if immunosuppressive drug treatment and immunodeficiency could represent predisposing factors for viral infection and severe SARS-CoV-2 complications is still not clear [2]. Alemtuzumab is an anti-CD52 humanized monoclo-

Lorenzo Saraceno

lorsaraceno@gmail.com

1 ASST Grande Ospedale Metropolitano Niguarda, Department of Neurosciences, Multiple Sclerosis Center, Piazza dell'Ospedale Maggiore 3, 20162 Milan, Italy

2 ASST Grande Ospedale Metropolitano Niguarda, Infectious Diseases Unit, Piazza dell’Ospedale Maggiore 3, 20162 Milan, Italy nal antibody approved for the treatment of highly active MS. By antibody-dependent cell-mediated cytotoxicity induction and complement activation, it leads to circulating $\mathrm{T}$ and memory $\mathrm{B}$ cell rapid profound depletion. $\mathrm{T}$ and $\mathrm{B}$ cell repopulation by precursor cells is respectively completed within about 2-3 years and 7 months after drug administration. After the treatment, a mild to moderate increase of upper and lower respiratory tract infections is reported [3], especially in the first month. However, innate immunity is mainly preserved since macrophages, natural killer cells and neutrophils have low CD52 expression [3].

\section{Case report}

In August 2018, a 28-year-old woman was referred to our Department for the subacute onset of impaired sensation in all four limbs secondary to a cervical myelitis. Magnetic resonance imaging (MRI) studies showed multiple brain 
and spinal cord inflammatory demyelinating lesions $(>12)$. Gadolinium enhancement was evident in a cervical lesion and in four periventricular plaques. In presence of cerebrospinal fluid oligoclonal bands intrathecal synthesis, MS was diagnosed according to McDonald 2017 criteria. After the patient relapsed with a dorsal myelitis 2 months later, presenting multiple new brain and spinal cord lesions, she was treated with alemtuzumab in November 2018 and 12 months later. To date, no relapses or MRI disease activity were reported after alemtuzumab starting. She showed clinical improvement and reduction of her Expanded Disability Status Scale (EDSS) from 2.5 to 1.0. In March 2020, 4 months after alemtuzumab II cycle, while she was leukopenic and lymphopenic (white cells $2.73 \times 10^{9} / \mathrm{L}$; lymphocytes $630 \mathrm{cell} / \mathrm{microL} ; \mathrm{CD} 3+408 \mathrm{cell} / \mathrm{microL} ; \mathrm{CD} 19+119$ cell/microL, CD4+/CD8 + 0.53), her whole family was coinfected by SARS-CoV-2 virus. Her 69-year-old father, with no comorbidities, was hospitalized for 2 weeks due to a bilateral interstitial pneumonia causing acute respiratory failure. He had hyperpyrexia $\left(38.5^{\circ} \mathrm{C}\right)$, headache and muscle pain. Her 67-year-old mother presented hyperpyrexia, dysgeusia, hyposmia and widespread muscle pain for a week. Her 25-year-old partner had hyperpyrexia up to $39{ }^{\circ} \mathrm{C}$ for 4 days, cough, chest pain, ageusia, hyposmia, widespread muscle pain and extreme fatigue. Our 30-yearold MS patient presented only mild cough, mild retrosternal chest pain and hyposmia for 3-4 days, without even hyperpyrexia. They all had positive nasopharyngeal swabs during the acute viral disease and then positive serological tests. Our patient developed symptoms on March 28. The first nasopharyngeal swab for SARS-CoV-2 RNA performed 4 days later was positive. Swab negativization was detected after 14 days. The $\operatorname{IgG}$ serologial test made 6 weeks later showed positive anti-S1 and S2 SARS-CoV-2 IgG antibodies with a titre of 26 (positive $\geq 15$ ). Brain and spinal cord MRI repeated 4 months after the infection confirmed stable disease.

Conclusions Real word data of SARS-CoV-2 infection in MS patients treated with disease modifying treatments (DMTs) are limited. Immunodepleting DMTs could represent a risk factor for virus infection and/or prolong disease duration by delaying viral elimination. According to actual recommendations made by Italian Society of Neurology (SIN) and Association of British Neurologists $(\mathrm{ABN})$ [2], the use of cladribine and especially alemtuzumab for MS is today discouraged during the SARS-CoV-2 pandemic. Since current data are too limited to evaluate the risk of severe SARS-CoV-2 disease after treatment, alemtuzumab should be started only when this risk is counterbalanced by significant benefits [2]. We report the case of a whole family coinfected by SARS-
CoV-2. Our MS patient presented mild infection symptoms despite leukopenia and lymphopenia following alemtuzumab II cycle. Not only she had no complications, but it is remarkable to report that she also presented milder COVID-related signs and symptoms as compared to her coinfected relatives. As shown in Table 1 other 11 cases of SARS-CoV-2 infection following alemtuzumab administration have been reported in literature [3-9]. As for our patient, none of them had complications or severe disease. Besides case reports, important data could also be obtained by pharmacovigilance reports and real-world registries. A total of 117 confirmed SARS-CoV-2 infections in patients previously treated (33 patients $<12$ months) with alemtuzumab have been reported to pharmacovigilance [10]. Among confirmed cases, no deaths were reported and in those recently treated ( $<6$ months), only one patient was hospitalized [10]. Some real-world registries did not find significant association between DMTs and both SARS-CoV-2 risk and clinical disease severity $[11,12]$. Other larger registries instead observed an increased frequency of severe SARS-CoV-2 disease only in patients undergoing antiCD20 therapies, confirming no increased SARS-CoV-2 risk in alemtuzumab-treated patients [8, 13, 14]. Entering the respiratory epithelial cells, SARS-CoV-2 virus lead to the activation of innate immune cells as macrophages, stimulating the production of cytokines and chemokines which, in their turn, recall the adaptive immune response represented by $\mathrm{T}$ cells in the lungs $[1,15]$. In severe SARS-CoV-2 disease, this crosstalk between innate immunity and $\mathrm{T}$ cells is unregulated and ultimately conduce to the massive amplification of cytokine production, T-cell overactivation, excessive infiltration and tissue damage [15]. It is possible to speculate that in young MS patients, a moderate immunosuppression could mitigate this pathological crosstalk between innate immune system and $\mathrm{T}$ cells, so preventing $\mathrm{T}$ cell overactivation and hyperinflammation that may lead to severe SARSCoV-2 disease. In comparison with $\mathrm{T}$ cells, repopulation of naive $\mathrm{B}$ cells is more rapid starting within 3 months after alemtuzumab treatment [9]. A blunted humoral response to SARS-Cov2 is expected during the maximal B cell depletion period. All 5 patients reported in literature who performed serological tests developed protective antibodies [Table 1]. However, only one was infected by SARS-CoV-2 virus within 3 months after alemtuzumab treatment. Furthermore, as in our patient, antibody titers were usually low, suggesting the need of a booster vaccine to maintain a protective immunity in the long-term period. Further studies are necessary to understand the complex relationship among coronavirus, immune system and immunosuppressant drugs. 


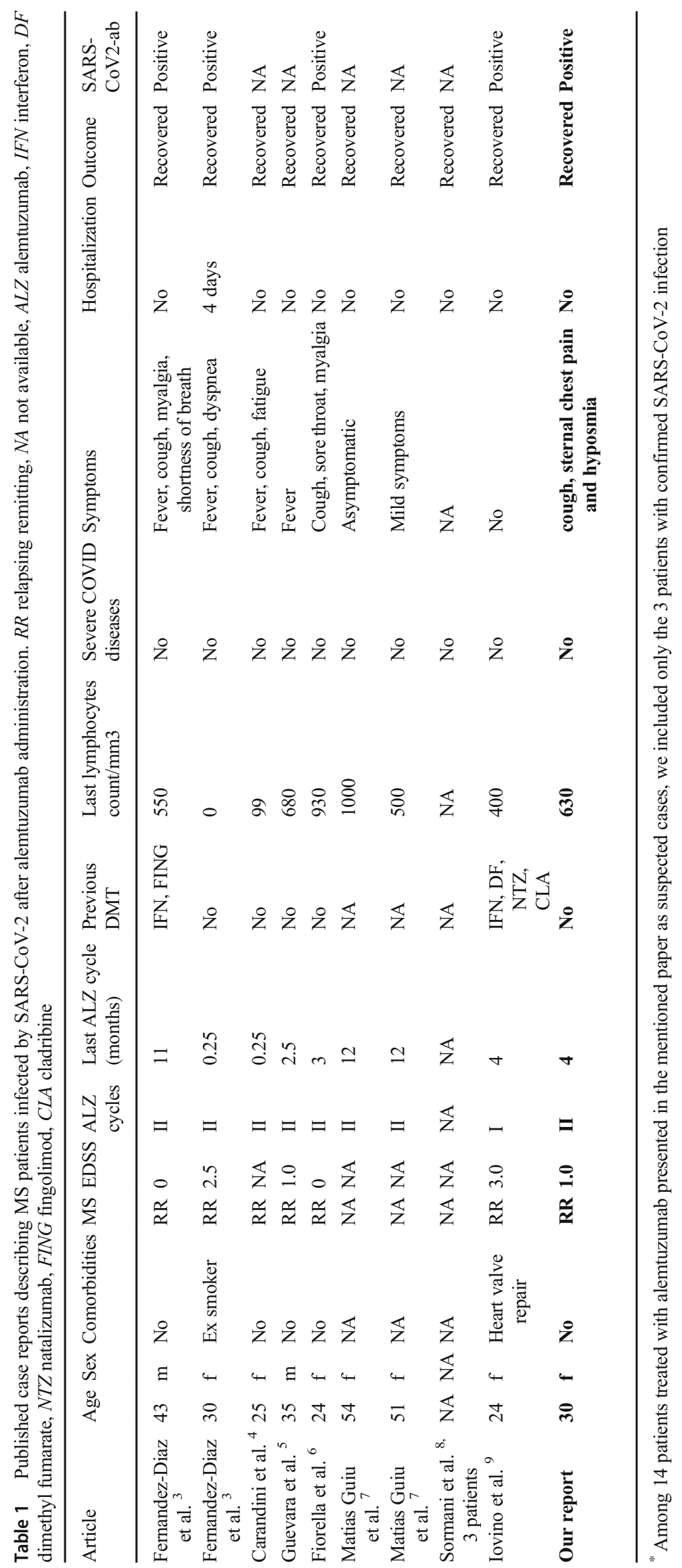


Acknowledgements We thank the patient for her contribution in providing clinical information.

Availability of data and material Original data and materials are available in the Center archive.

Author contribution LS, ELS, MRM, MCM and AP took care of the patient. LS, ELS and AP were the principal investigators and wrote the paper. ECA and AP reviewed the draft and approved the final version of the manuscript.

\section{Declarations}

Ethical approval None.

Consent for publication Informed consent was obtained from the patient.

Competing interests The authors declare no competing interests.

\section{References}

1. Zhou F, Yu T, Du R et al (2020) Clinical course and risk factors for mortality of adult in patients with COVID-19 in Wuhan, China: a retrospective cohort study. Lancet 395(10229):1054-1062. https:// doi.org/10.1016/S0140-6736(20)30566-3

2. Giovannoni G, Hawkes C, Lechner-Scott J et al (2020) The COVID-19 pandemic and the use of MS disease-modifying therapies. Mult Scler Relat Disord 39:102073. https://doi.org/10.1016/j. msard.2020.102073

3. Fernández-Díaz E, Gracia-Gil J, García-García JG et al (2020) COVID-19 and multiple sclerosis: a description of two cases on alemtuzumab. Mult Scler Relat Disord 45:102402. https://doi.org/ 10.1016/j.msard.2020.102402

4. Carandini T, Pietroboni AM, Sacchi et al (2020) Alemtuzumab in multiple sclerosis during the COVID-19 pandemic: a mild uncomplicated infection despite intense immunosuppression. Mult Scler: 1352458520926459. https://doi.org/10.1177/1352458520926459

5. Guevara C, Villa E, Cifuentes M, Naves R, Grazia J (2020) Mild COVID-19 infection in a patient with multiple sclerosis and severe depletion of T-lymphocyte subsets due to alemtuzumab. Mult Scler Relat 44:102314. https://doi.org/10.1016/j.msard.2020.102314

6. Fiorella C, Lorna G et al (2020) COVID-19 in a multiple sclerosis (MS) patient treated with alemtuzumab: insight to the immune response after COVID. Multiple Sclerosis and Related Disorders 46:102447. https://doi.org/10.1016/j.msard.2020.102447

7. Matías-Guiu J, Montero-Escribano P, Pytel V, Porta-Etessam J, Matias-Guiu JA (2020) Potential COVID-19 infection in patients with severe multiple sclerosis treated with alemtuzumab. Mult Scler Related Disorders 44:102297. https://doi.org/10.1016/j.msard. 2020.102297

8. Sormani MP, De Rossi N, Schiavetti I et al (2021) Diseasemodifying therapies and coronavirus disease 2019 severity in multiple sclerosis. Ann Neurol. https://doi.org/10.1002/ana.26028

9. Iovino A, Olivieri N, Aruta F, Giaquinto E, Ruggiero L, Spina E, Tozza S, Manganelli F, Iodice R (2021) Alemtuzumab in Covid era. Mult Scler Related Disorders 51:102908. https://doi.org/10. 1016/j.msard.2021.102908

10. Giovannoni G, Boster AL, Berkovich R et al (2021) Cases of COVID-19 in Alemtuzumab-treated patients with MS: pharmacovigilance report. American Academy of Neurology

11. Dalla Costa G, Leocani L, Montalban X, Guerrero AI, Sørensen PS, Magyari M, Dobson RJB, Cummins N, Narayan VA, Hotopf M, Comi G, on behalf of the RADAR-CNS consortium (2020) Realtime assessment of COVID-19 prevalence among multiple sclerosis patients: a multicenter European study. Neurol Sci 41(7):16471650. https://doi.org/10.1007/s10072-020-04519-x

12. Louapre C, Collongues N, Stankoff B, Giannesini C, Papeix C, Bensa C, Deschamps R, Créange A, Wahab A, Pelletier J, Heinzlef O, Labauge P, Guilloton L, Ahle G, Goudot M, Bigaut K, Laplaud DA, Vukusic S, Lubetzki C et al (2020) Clinical characteristics and outcomes in patients with coronavirus disease 2019 and multiple sclerosis. JAMA Neurol 1(77(9)):1079-1088. https:// doi.org/10.1001/jamaneurol.2020.2581

13. Reder AT, Centonze D, Naylor ML, Nagpal A, Rajbhandari R, Altincatal A, Kim M, Berdofe A, Radhakrishnan M, Jung E, Sandrock AW, Smirnakis K, Popescu C, de Moor C (2021) COVID-19 in patients with multiple sclerosis: associations with disease-modifying therapies. CNS Drugs 35(3):317-330. https:// doi.org/10.1007/s40263-021-00804-1

14. Safavi F, Nourbakhsh B, Azimi AR (2020) B-cell depleting therapies may affect susceptibility to acute respiratory illness among patients with multiple sclerosis during the early COVID-19 epidemic in Iran. Mult Scler Relat Disord.43:102195. doi: https://doi.org/ 10.1016/j.msard.2020.102195, 43, 102195

15. Biying H, Shaoying H, Lianghong Y (2021) The cytokine storm and. COVID-19- J Med Virol 93(1):250-256. https://doi.org/10. $1002 / \mathrm{jmv} .26232$

Publisher's note Springer Nature remains neutral with regard to jurisdictional claims in published maps and institutional affiliations. 